\title{
Vitiligo patients have low risk for skin cancer
}

Published online: 16 November 2012

(C) Springer Healthcare 2012

medwireNews: Evidence suggests that patients with vitiligo have a reduced risk for both melanoma and nonmelanoma skin cancer.

Recent studies have shown a decreased genetic susceptibility to melanoma in patients with vitiligo, but prevalence of skin cancer in people with this disorder has not been previously studied.

Hansje-Eva Teulings (University of Amsterdam, the Netherlands) and colleagues carried out a survey of 1307 patients with nonsegmental vitiligo and 788 controls without the condition to assess their lifetime rates of skin cancer. The mean age of the participants was 60.5 years and all skin types were represented, with skin types 2 and 3 being the most common.

As reported in the British Journal of Dermatology, the team found that seven people in the vitiligo group and 12 in the control group had a prior diagnosis of melanoma, translating to a significant $68 \%$ reduced risk for melanoma in vitiligo patients versus controls after controlling for skin cancer risk factors such as skin type, number of nevi, and sun exposure.

Similarly, more controls had a history of basal cell carcinoma (BCC) and squamous cell carcinoma (SCC) than people in the vitiligo group, at 47 and four versus 37 and five people, respectively. This translated to a significant $72 \%$ reduction in risk for nonmelanoma skin cancer (BCC and SCC) in people with vitiligo versus controls after adjusting for confounders.

Of note, prior phototherapy treatment of 911 patients with vitiligo did not appear to increase their risk for skin cancer compared with controls.

"The antimelanocyte immune response in patients with vitiligo may be responsible for the observed decrease in melanoma lifetime prevalence," suggest Teulings and team.

"These results are also consistent with recently published genetic studies showing the likelihood of the mutual exclusiveness of vitiligo and melanoma, as the major alleles of SNPs in the TYR gene are associated with vitiligo, and the minor alleles with melanoma," they add.

By Helen Albert, Senior medwireNews Reporter

\section{Reference}

Br J Dermatol 2012; Advance online publication 